\section{TECNOLOGÍA DE HERRAMIENTAS ROTATIVAS DURANTE EL BRONCE FINAL ATLÁNTICO. EL DEPÓSITO DE VILLENA}

\author{
ROTARY MOTION TOOLS \\ TECHNOLOGY IN ATLANTIC FINAL \\ BRONZE AGE. THE VILLENA GOLD \\ HOARD
}

\section{BARBARA R. ARMBRUSTER (*) ALICIA PEREA (**)}

\section{RESUMEN}

El estudio de los brazaletes del tesoro de Villena es el punto de partida de una investigación en equipo que ha tenido como resultado la constatación de un estadio tecnológico más avanzado del que hasta ahora se pensaba para la metalurgia del Bronce Atlántico en la Península Ibérica. Se demuestra la práctica de una sofisticada técnica de fundición a la cera perdida en oro y la existencia del torno de eje horizontal. Finalmente planteamos un modelo interpretativo para este importante depósito y situamos en un marco de cronología relativa toda una serie de hallazgos de orfebrería del suroeste peninsular que hasta la fecha se encontraban descontextualizados.

\footnotetext{
ABSTRACT

The analysis of the Villena goldhoard bracelets is the starting point for a team research which has showed the existence of a technological stage which was more develo-

(*) Universidad de Tübingen. Programa de Arqueometalurgia de la Fundación Volkswagen.

(**) Departamento de Prehistoria, Centro de Estudios Históricos. Consejo Superior de Investigaciones Científicas. Serrano, 13. 28001 Madrid.

El artículo fue remitido en su versión final el 19-IV-94.
}

ped than we thought for the Atlantic Bronze Age in the Iberian Peninsula. The use of a complex lost wax casting gold technique and the existence of a horizontal rotary axis lathe are demonstrated. Finally we put forward an explanation for this important hoard and we stablish a chronological frame work framework for a series of gold finds coming from the southwest of the Iberian Peninsula.

Palabras clave: Metalurgia. Tecnología. Oro. Cera perdida. Torno de eje horizontal. Depósito. Bronce Atlántico.

Key words: Metallurgy. Technology. Gold. Lost wax. Horizontal axis lathe. Hoard. Atlantic Bronze Age.

\section{INTRODUCCIÓN}

\subsection{Experiencia de un trabajo en equipo}

En el ámbito de la investigación arqueológica es ya imprescindible el planteamiento del trabajo desde una perspectiva interdisciplinar. El equipo se beneficia de la multiplicidad de enfoques y la potencialidad intelectual acumulativa de cada uno de sus miembros. Estos, tienen 
sus foros de discusión específica en las revistas especializadas y en los Congresos y Symposia, pero, en general, no trascienden la mera exposición pública y reafirmación personal o institucional; en definitiva, cumplen con la labor de difusión, pero en contadas ocasiones con la de debate.

Nuestro planteamiento al abordar este estudio fue precisamente beneficiarnos del trabajo en equipo y huir de la reafirmación. El nuestro es, por tanto, un equipo interdisciplinar, independiente y no institucional. Tuvo su origen en la divergencia de opiniones y la discusión científica, y se enriqueció con la diversidad de procedencia y formación académica de sus dos componentes, e incluso con la lingüística.

Llevar a la práctica estos planteamientos no está exento de ciertos inconvenientes. En primer lugar, huir de la reafirmación supone adoptar una actitud de autocrítica y permeabildad teórica y metodológica. Nuestro origen académico se situaba entre la tradición clásica germana y la postmodernidad anglosajona matizada por el filtro peninsular. En el caso de B. Armbruster enriquecido por la práctica del oficio de orfebre, una gran ayuda a la hora de descender del limbo intelectual y pisar tierra sórdida y firme.

La independencia se paga con la escasez de medios y la falta de continuidad, aunque se suple con el entusiasmo. Finalmente, la diversidad lingüística requiere un esfuerzo suplementario en la función comunicativa, base fundamental del entendimiento y requisito imprescindible de un equipo, pero obliga a esclarecer conceptos y organizar ideas. Esperamos que haya merecido la pena.

\subsection{Metodología}

La identificación de huellas de trabajado sobre metales para la reconstrucción de técnicas y herramientas es una metodología que empezó a aplicarse de manera sistemática en Arqueología hacia los años 70 (por ejemplo: Lowery, Savage, Wilkins, 1971).

La base teórica de este método estriba en lo que L. Biek (1963: 54 y ss.) denominó "aproximación vertical". Existe una gran cantidad y variedad de información en un objeto según vayamos ganando en aumentos, desde la observación a ojo desnudo, hasta el examen con microscopios de alta resolución (por ejemplo Olsen, 1988; Perea, 1990). No es lo mismo el dato que aporta un estudio con lupa binocular de 2 aumentos, que el que podamos obtener con una metalografía de la misma pieza a 120 . Las posibilidades son enormes y sólo se ven limitadas por la disponibilidad de un equipo adecuado y el estado de conservación de las piezas.

En el caso del depósito de Villena contábamos con un condicionante previo: las piezas debían ser examinadas en el Museo local y con el equipo que nosotras trasladásemos a él. Creímos suficiente una lupa binocular, portátil, con capacidad hasta de 2 aumentos, y sendos equipos de macrofotografía para la documentación gráfica de las incidencias tecnológicas.

Cuando se acomete un "trabajo de Museo" de este tipo es inevitable el olvido de alguna observación concreta que, con el paso del tiempo, se vuelve imprescindible a ojos del investigador que se encuentra ya lejos de su objeto de deseo. Para evitarlo adoptámos la elemental solución de tomar pequeños moldes de silicona en aquellas zonas de las piezas que creímos más interesantes. Estos moldes, con su correspondiente identificación grabada, reproducen micrométricamente la superficie del metal, de manera que pueden ser examinados bajo cualquier instrumento óptico como si fuera un negativo del original (ver por ejemplo: Lowery, Savage, 1972; Larsen, 1987).

Una vez finalizada la fase de observación, planteamos nuestra hipótesis tecnológica y pasamos a la de experimentación. Con ello tratábamos de comprobar el grado de probabilidad que tenía nuestra hipótesis de cumplirse, reproduciendo la técnica en las condiciones supuestamente originales. Consideramos imprescindible la metodología experimental a la hora de abordar el estudio de técnicas, que por cuestiones económicas o de otro tipo han dejado de practicarse en el entorno tecnológico actual (por ejemplo: Schiffer, Skibo, 1987). En este sentido defendemos igualmente para este tipo de investigación el enfoque y la colaboración que aportan otras disciplinas y profesiones. La Etnología y la Etnoarqueología son de especial interés al ponernos en contacto con artesanos y técnicas que han desaparecido de nuestro entorno (Armbruster, 1992, 1993b); la filología nos permite interpretar las fuentes antiguas y medievales; la iconografía, representaciones figuradas de herramientas y procesos técnicos; arqueometría, macro y microscopía, etc. son otras de las técnicas analíticas que deben incor- 
porarse de manera sistemática a la reconstrucción de nuestro pasado tecnológico.

Finalmente, los datos técnicos y arqueológicos han sido la base para la elaboración de dos modelos explicativos. El primer modelo concierne exclusivamente al depósito de Villena. No se ha intentado solventar todos los problemas de este complejo hallazgo, sino de plantear un marco interpretativo más coherente con nuestro conocimiento actual.

El segundo modelo, más general, es conclusión y síntesis de la trayectoria de la metalurgia del oro durante el Bronce Final en la fachada atlántica peninsular.

\subsection{El depósito de Villena}

La vega de Villena (Alicante), recorrida en su último tramo por el río Vinalopó, es un amplio corredor que pone en contacto tres ámbitos geográficos diferentes: el Mediterráneo, Andalucía y la Meseta.

Los recursos naturales (Soler, 1987: 11-15) y su situación estratégica (Rubiera, 1985; RuizGálvez, 1989: 54-55), así como una peculiar e intensa historia de trabajos de campo arqueológicos, explican la densidad de yacimientos y hallazgos fortuitos conocidos en la zona, cuyo descubrimiento se debe, casi en exclusiva, a la dedicación de Don J.M. Soler a lo largo de toda una vida (Soler, 1987, donde se recoge su bibliografía anterior).

Entre Octubre y Noviembre de 1963 se produce el hallazgo de un depósito en una rambla utilizada como gravera, compuesto por $9 \mathrm{~kg}$. 112 gr. de oro, entre brazaletes, elementos de vajilla y posibles restos de guarniciones de armas, además de algunas piezas en plata, dos de hierro y una que contenía ámbar. Las primeras piezas de oro -unos brazaletes- se encontraron casualmente, pero el grueso del llamado "tesoro de Villena" apareció en el curso de las excavaciones efectuadas en el lugar del hallazgo inicial (Soler, 1965). No existe, sin embargo, indicio de contexto arquitectónico o de otro tipo, salvo el propio de la tosca vasija que lo contenía en ordenada disposición.

Unos meses antes, y a unos $6 \mathrm{~km}$. del anterior, había aparecido fortuitamente otro depósito de oro, más modesto (Soler, 1965). En este caso las piezas se encontraron de manera dispersa y desordenada durante los trabajos de desmonte en una cantera de yeso. El lugar estaba próximo a los restos de las viviendas del poblado de Cabezo Redondo, pero no en su interior, y a poca profundidad de la superficie. Por oposición al anterior, este hallazgo fue conocido como "tesorillo de Cabezo Redondo". Ambos se conservan en la actualidad en el Museo J.M. Soler de Villena (1).

Las circunstancias temporales y espaciales de los dos hallazgos han condicionado en gran medida su estudio, puesto que tanto uno como otro se han asociado indefectiblemente, y sin cuestionamiento, al poblado de Cabezo Redondo y, en consecuencia, se han considerado metodológicamente como un único hallazgo.

\section{HISTORIA DE LA INVESTIGACIÓN Y NUEVAS ORIENTACIONES}

\subsection{Conclusiones obtenidas con una metodo- logía tradicional}

La investigación sobre Villena se ha centrado en dos aspectos casi únicos: situación cronológica y técnica de fabricación de los brazaletes.

La cuestión cronológica se ha basado en argumentos tipológicos y paralelos formales. En la base de todo el razonamiento de Soler (1965) subyace, en primer lugar, la idea de coetaneidad entre "tesoro" y "tesorillo" (Soler, 43), y en segundo, la de una fabricación local dentro del poblado de Cabezo Redondo (Soler, 49) por lo que la fecha no puede en ningún caso anteceder o preceder a la de ocupación del yacimiento. Propone un momento en torno al año 1000 a.C., fase final de ocupación. Además, otros brazaletes conocidos de la misma tipología, como los portugueses de Estremoz y Portalegre, serían importaciones del taller villenense (Soler, 47 y 51). Este localismo se apoya en una forzada argumentación sobre la posible existencia de arenas fluviales auríferas en la zona (Soler, 1969; 1987: 151) y en los paralelos formales entre la cerámica del poblado y los cuencos del tesoro (Soler, 1965: 44).

Por su parte Almagro Gorbea (1974) institucionaliza el concepto "orfebrería tipo Villena" y defiende un origen centroeuropeo para los cuencos, mediterráneo para las botellas. En

(1) Agradecemos a D. J.M. Soler el permiso para examinar y fotografiar el depósito de Villena, las facilidades que en todo momento nos proporcionó, y sobre todo, su amable y cálida acogida. 
cuanto a los brazaletes, se decanta por una posible fabricación local, pero con un origen exterior, igualmente centroeuropeo. El ámbito de desarrollo cronológico para este tipo de orfebrería se prolonga entre el siglo IX y el VII a.C. En toda su argumentación subyace la influencia del ya clásico estudio de Blanco Freijeiro (1957) donde se definía la orfebrería hallstáttica.

Finalmente Ruíz-Gálvez (1992), junto con Schüle (1976), cree en la fabricación local de todo el conjunto, basándose ambos en que cuencos y botellas serían la traducción al metal de formas cerámicas de la cultura Cogotas I que están presentes en Cabezo Redondo. Para Ruíz-Gálvez la aparición de hierro en alguna de las piezas del depósito es determinante para su situación cronológica; el límite inferior se sitúa en el siglo VIII a.C. y el superior, más problemático, hacia el siglo XIII-XII a.C. en relación con un momento anterior a la desaparición del asentamiento de Cabezo Redondo y las cerámicas tipo Cogotas I.

El aspecto técnico ha sido otro de los temas polémicos y debatidos y se ha referido exclusivamente al problema planteado por los brazaletes con decoración de molduras, púas y calados. En este sentido nos parece importante destacar que entre todos los estudios publicados ninguno de ellos se ha basado en datos analíticos o en la observación de las piezas mediante algún tipo de ayuda óptica; incluso algunas de las opiniones emitidas, se hicieron sobre base documental exclusivamente fotográfica. Excepcionalmente, Blanco Freijeiro (1957) estudió el brazalete de Estremoz con una lupa binocular, pero su desconocimiento sobre tecnología del oro hizo que la interpretación fuera totalmente errónea.

La polémica comienza ya en 1912, cuando Reinach publica por vez primera el famoso brazalete de Estremoz. Se pueden resumir las distintas hipótesis emitidas en dos grandes grupos: aquellas que admiten el empleo de técnicas metalúrgicas avanzadas, como el moldeado o la soldadura, y las que no lo admiten. Entre las primeras Blanco Freijeiro (1957: 8, fig. 3) se decanta por el empleo de la soldadura para supuestos componentes individualizados, mientras que Russel (1954) apoya una técnica combinada de moldeado y soldadura por "percusión".

Entre las segundas se encuentra Soler (1965: 19) quien propone una combinación de batido sobre molde para las molduras y cincelado para púas y perforaciones. Por su parte Schüle (1976: 153 y ss.) defiende el tallado o conformado del metal en caliente, sin pérdida de material. Finalmente se pueden agrupar las hipótesis de Cardozo (1959: 24), Nicolini (1990: 19-21), y una de nosotras (Perea, 1991: 98; 1993: 24) con una técnica basada en el tallado y corte del metal a partir de un cilindro fundido.

Concerniente a los aspectos socioeconómicos del depósito, las interpretaciones han alternado entre su consideración como depósito de fundidor u orfebre (Soler, 1965; Almagro Gorbea 1974; Perea, 1991) y su consideración como tesoro o posesión personal (Tarradell, 1964; Soler, 1965; Maluquer, 1970). Recientemente Ruíz-Gálvez (1992: 232-236) ha realizado un sugerente estudio en donde relaciona la aparición de piezas de vajilla de oro con formas comunitarias de comida y bebida dentro del entorno social masculino, por ello, interpreta el depósito como la propiedad personal de un único individuo, siendo los brazaletes posibles formas de pago o tributo. En la base de su argumentación está la idea de la fabricación y utilización de estas piezas en la zona de Villena, muy en relación con el asentamiento de $\mathrm{Ca}$ bezo Redondo; los brazaletes encontrados en la fachada atlántica serían prueba de la llegada a esa zona de técnicas, y quizá mujeres, desde el levante peninsular.

\subsection{Nuevas propuestas de trabajo e hipótesis tecnológica}

En la publicación de una de nosotras sobre orfebrería prerromana (Perea, 1991) se abordó el espinoso tema de la técnica empleada en la fabricación de los brazaletes tipo Villena-Estremoz. El estudio se había realizado sobre la observación del ejemplar de Estremoz y un fragmento de procedencia dudosa, posiblemente León, conservados ambos en el Museo Arqueológico Nacional, ya que en el Museo de Villena no existían medios ópticos para el estudio de sus ejemplares. Entonces (Perea, 1991: 98-100) se descartó el empleo de técnicas como el fundido en molde y la soldadura por dos motivos, primero, porque las huellas observadas no encajaban con estas técnicas, y segundo, porque el ambiente tecnológico en el que había que encuadrar esta producción tampoco avalaba su empleo. Se tomó, por tanto, una variante de la hipótesis de Schüle (1976) como válida, dado

T. P., 51, nº 2, 1994 
que era la que mejor se podía ajustar a la realidad de lo observado. Y esa realidad eran huellas aparentemente de talla, sobre todo en la zona de púas del fragmento. Posteriormente, Perea (1993) creyó importante plantear la cuestión del artesanado; según su propuesta, debió existir un taller de orfebrería relacionado con los brazaletes de este tipo. Se trataría del primer taller identificable en la Península Ibérica.

Paralelamente, Armbruster había estudiado varios ejemplares portugueses del tipo VillenaEstremoz, conservados en el Museu Nacional de Arqueologia e Etnologia de Lisboa (Inventario 1993: 130-137, 140-143). Su reciente estancia en el Africa subsahariana, compartiendo los conocimientos artesanales de los orfebres de Mali y Burkina Faso, además de otros centros artesanales de Egipto, India y Sri Lanka, habían enriquecido y matizado su visión sobre la práctica de la orfebrería y la interpretación tecnológica prehistórica (Armbruster, 1992, 1993a, 1993b). Su hipótesis partía de la base de una mayor capacidad técnica de los orfebres de la Edad del Bronce, y de una errónea interpretación de las huellas de trabajado por parte de los autores que habían tocado el tema.

Efectivamente, en nuestras discusiones previas y durante el estudio realizado, quedó patente que Perea había tomado la imagen del espejo por la real. Esto es, lo que creía huellas del trabajo directo sobre el metal, eran huellas dejadas por las herramientas sobre el molde de cera que posteriormente iban a reflejarse de manera fiel en el negativo metálico. Otro de los errores cometido fue la elección del ejemplar de Estremoz como objeto de estudio ya que su perfecta fabricación y acabado lo invalidan prácticamente para este tipo de estudios, donde las piezas defectuosas muestran más claramente huellas y procesos difíciles de identificar en su perfección.

El empleo de herramientas y técnicas hasta ahora no identificadas en la Edad del Bronce supuso un cambio de actitud y de visión hacia el análisis arqueológico y hacia los parámetros tecnológicos establecidos por la investigación tradicional. Intentamos una investigación autocrítica, para la que tuvimos que prescindir de todos los presupuestos anteriores, y partir prácticamente de cero. Ello nos obligó a analizar el depósito de Villena aislándolo del entorno cultural de su zona de hallazgo, para situarlo en un entorno tecnológico coherente con su realidad. Hemos sido conscientes de los riesgos que ello implica.

\section{ASPECTOS FORMALES Y TECNOLÓGICOS}

Debido a las nuevas investigaciones que hemos llevado a cabo, creemos ineludible definir nuevamente la llamada orfebrería "tipo Villena".

Este tipo supone los siguientes aspectos formales: objetos perfectamente cilíndricos, en su origen, con alguno de los siguientes elementos decorativos: molduras de diferentes tamaños y grosores que ocasionalmente pueden presentar incisiones o cortes paralelos; perforaciones rectangulares u ovaladas en series lineales; púas piramidales o cónicas igualmente alineadas en series.

Con respecto a los aspectos tecnológicos, el tipo implica el empleo de instrumentos rotativos para la fabricación de moldes en cera y para el acabado de la pieza metálica, ello demuestra una tecnología avanzada de la fundición a la cera perdida.

La definición de este tipo excluye, por tanto, todas las piezas del depósito de Villena que no sean los brazaletes. Queremos hacer hincapié en ello porque la denominación "tipo Villena" puede inducir a error por generalización; por ello, creemos más adecuada la denominación "tipo Villena-Estremoz" (tipo V/E).

El tipo $\mathrm{V} / \mathrm{E}$ incluye piezas anulares que desde el punto de vista actual denominaríamos brazaletes y anillos.

Existen otra serie de piezas que presentan alguno de los aspectos formales señalados en el tipo V/E, y algunas de las técnicas apuntadas, pero no todas, y que consideramos como productos derivados o evolucionados a partir del tipo, y para cuya fabricación ha sido necesaria la transmisión del know how mediante algún tipo de contacto personal. Estas piezas las denominaremos "derivadas" o "evolucionadas" sin que ello suponga mayor complejidad, avance tecnológico, o pericia artesanal. Solamente en un caso (Monte da Saia, Braga) hemos encontrado una pieza que podría calificarse de "imitación", entendiendo por ello el intento de reproducir el tipo V/E sin que haya existido una transmisión personal de la tecnología.

Creemos que la transmisión tecnológica de procesos complejos sólo es posible mediante el contacto personal entre artesanos. La naturaleza del conocimiento tecnológico, simple o complejo, se basa en tres principios (Schiffer, Skibo, 1987): a) comportamiento tecnológico, o 


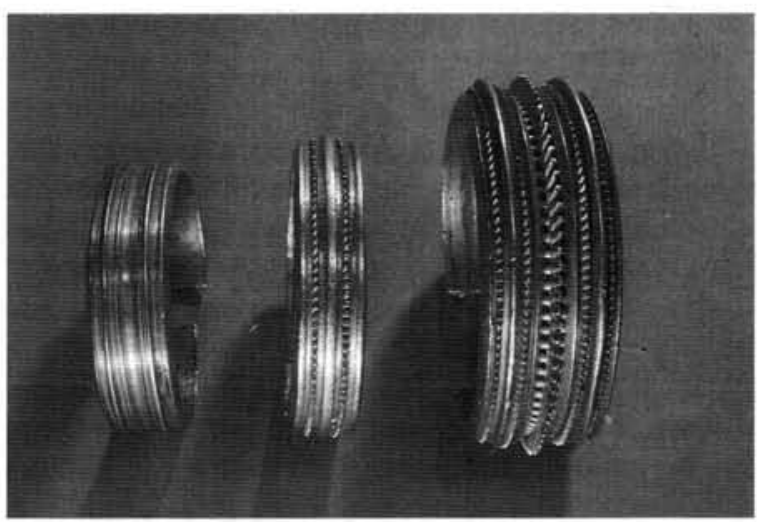

Lám. I. Algunas variantes de los brazaletes del depósito de Villena.

proceso de transformación de la materia prima en producto elaborado; b) aprendizaje, o método de transmisión de ese proceso mediante imitación, contacto verbal o autoaprendizaje mediante prueba y error; y c) tecno-ciencia, o conocimiento de los principios científicos que rigen el comportamiento tecnológico. En la tecnología V/E no existía tecno-ciencia, y creemos que su transmisión se realizó mediante aprendizaje, por visualización del comportamiento tecnológico y no únicamente por visualización del producto acabado.

\subsection{Los brazaletes del depósito de Villena.}

\section{Formas y secciones.}

Los 28 brazaletes del tesoro de Villena (Lám. I) forman una serie de combinaciones posibles entre los elementos antes mencionados, molduras, púas y perforaciones: modelo plano-convexo; modelo combinado con una linea de perforaciones entre dos aros plano-con-

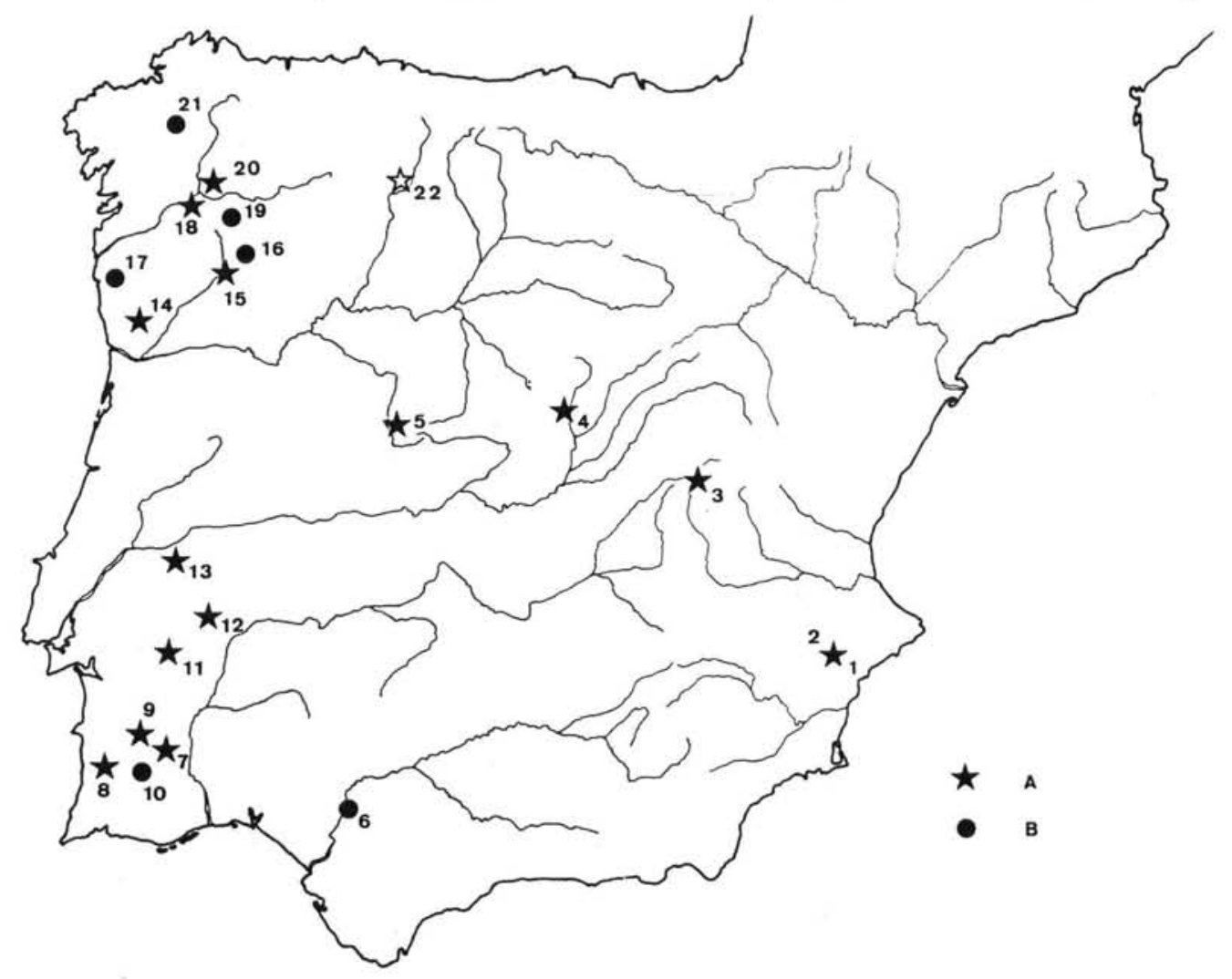

Fig. 1. A: Hallazgos de piezas tipo Villena-Estremoz. B: Hallazgos de piezas derivadas o evolucionadas. 1: Villena (Alicante). 2: Cabezo Redondo (Villena, Alicante). 3: Abía de la Obispalía (Cuenca). 4: La Torrecilla (Getafe, Madrid). 5: El Torrión (Navamorales, Salamanca). 6: El Carambolo (Camas, Sevilla). 7: Trindade (Beja). 8: Colos (Beja). 9: Aljustrel (Beja). 10: Beja (distrito). 11: Evora. 12: Estremoz (Evora). 13: Portalegre (Evora). 14: Cantonha (Penha, Braga). 15: Chaves (Vila Real). 16: Lebuçao (Vila Real). 17: Monte da Saia (Braga). 18: Toén (Orense). 19: Urdiñeira (A Gudiña, Orense). 20: Orense (provincia). 21: Mellid (La Coruña). 22: León (provincia?). No se han incluido dos ejemplares de procedencia desconocida en el Museo Soares dos Reis de Oporto, ni el brazalete n 16.853 del Museo Arqueológico Nacional de Madrid. 
vexos; combinaciones más complejas entre los distintos elementos (Soler, 1965: 14, fig. 4: Perea, 1991: 99, fig. 6). Estas series reflejan de manera simplificada los pasos sucesivos necesarios en la fabricación del modelo de cera; lo mismo que las secciones. Contrariamente a lo que hasta ahora se pensaba los brazaletes de sección plano-convexa también fueron fabricados con la técnica que se describe a continuación. Conocemos un total de 58 piezas tipo V/E (Fig. 1), de las que 47 son brazaletes, seis de sección planoconvexa, y cuarenta con molduras, púas y perforaciones. De estos 47 brazaletes, siete aparecen cerrados. Además, existen 11 anillos cerrados (Armbruster, 1993a: 276). En un estudio reciente se estableció la clara pertenencia del brazalete de "Orense" al tipo V/E, que hasta la fecha se había clasificado como una imitación trabajada en lámina.

\section{Huellas de herramientas y uso}

Todos los brazaletes se fundieron a la cera perdida, y la prueba se encuentra en las huellas evidentes de una estructura de fundición en la superficie, tanto en el interior como el exterior, en las púas y en las molduras.

Mediante las huellas que las herramientas dejan sobre la superficie se pueden deducir los métodos de fabricación y los útiles empleados. La perfecta simetría de las molduras nos obliga a suponer el empleo de un instrumento rotativo para la fabricación del modelo en cera; además, son visibles huellas de pulido que prueban que los brazaletes se retocaron en un torno tras la fundición. Otro indicio del empleo del torno es la perfecta circularidad de los cilindros que se puede observar en los ejemplares de Estremoz, Evora y La Torrecilla, conservados en su forma original cerrada. En cuanto al torno utilizado, tendríamos que imaginar una herramienta sencilla, con eje horizontal que rota mediante un arco o accionamiento de cuerda.

También las huellas de pulido que aparecen en las púas cónicas están indicando el empleo de una broca, o herramienta con eje vertical y punta hueca, que podría reconstruirse como un sencillo taladro accionado a cuerda o arco, con una cabeza donde las púas aparecen en negativo.

\section{Las singularidades de los tipos V/E: el brazalete abierto de Cantonha, Guimaraês}

Este brazalete (Lám. II) es una pieza extraordinaria dentro del tipo V/E (Inventario, 1993:

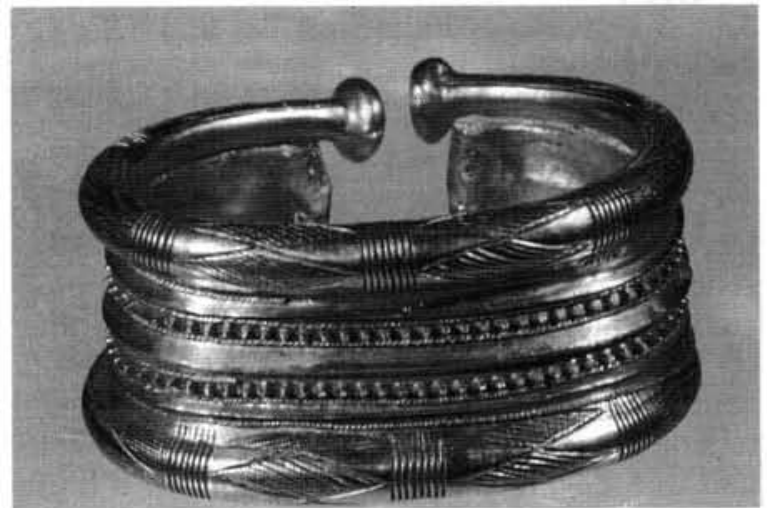

Lám. II. Brazalete de Cantonha, Guimaraês.

140-143), que además presenta una técnica de unión raramente empleada, y que podría describirse como un antecedente de la soldadura. El brazalete se compone de una parte central que responde por entero al tipo $\mathrm{V} / \mathrm{E}$, con molduras y púas; dos brazaletes exteriores, macizos, con decoración incisa, del tipo Sagrajas/Berzocana (Almagro Gorbea, 1974); y seis hilos de sección cuadrada torsionados, similares a los fragmentos que aparecen en el depósito de Sagrajas, Badajoz. El orfebre montó los diferentes elementos mediante la técnica del "casting-on" (fusión o fundición adicional). Los dos brazaletes exteriores, de sección circular, presentan unos extremos engrosados, añadidos en una fundición adicional. La decoración, cincelada sin levantamiento de viruta, es geométrica a base de rombos reticulados y líneas paralelas; este mismo tipo de decoración se imitó posteriormente, con otro cincel, en la zona de fusión adicional, entre el cuerpo central y los brazaletes exteriores. Esto prueba que los brazaletes presentaban una decoración incisa con anterioridad a que el orfebre uniera los diferentes elementos del conjunto.

Todavía hoy se debate la fecha del brazalete de Cantonha y de los torques macizos (siglos XIII a III a.C.), con diferentes propuestas que oscilan entre el siglo VIII y el I a.C. (Kalb, 1991: 188). Las toscas uniones trabajadas mediante la técnica del "casting-on" nos inducen a pensar que el brazalete de Cantonha quedó montado en el Bronce Final. En el torques doble de Sagrajas se empleó una técnica de unión parecida; y según él estado actual de nuestros conocimientos, las soldaduras de inicios de la Edad del Hierro son de realización más fina. 


\subsection{Evolución e "imitación" del tipo V/E.}

Los brazaletes que se relacionan a continuación pueden describirse como evoluciones o imitaciones del tipo V/E. Se diferencian en que no presentan todas las características formales y técnicas que definen el tipo, sino una o varias. Además, presentan elementos técnicos y tipológicos de ámbitos culturales limítrofes geográfica o temporalmente, lo que puede ser prueba de contactos interculturales, con o sin transferencia tecnológica. También denotan la amplia identidad que llegan a alcanzar la tradición e imitación de determinados elementos formales y decorativos, y el significado simbólico de objetos en metales preciosos, dentro de los sistemas de comunicación no verbales. Pese a los profundos cambios en la orfebrería peninsular bajo influencia orientalizante durante la Edad del Hierro, los elementos estilísticos de la Edad del Bronce se mantienen, perfeccionados y combinados con otros orientalizantes, como nos muestra el ejemplo de los brazaletes cilíndricos de El Carambolo.

- Monte da Saia, Braga (Cardozo, 1975). Cilindro cerrado, fragmentado, forma ondulada forjada, decoración a punzón. Bronce Final.

Este brazalete cerrado presupone la existencia de contactos sin transmisión del know-how puesto que recuerda el tipo V/E por su forma cilíndrica, y su organización en bandas. Sin embargo, un examen de detalle muestra una fabricación muy distinta. Se forjó a partir de una barra anular hasta conseguir un cilindro laminar. Las molduras están repujadas desde el interior. Con un punzón de cabeza circular se marcaron varias lineas de circulos que parecen imitar la decoración de púas de los tipos V/E.

- Mellid, La Coruña (Pingel, 1992: n 71, lám. $18,9)$. Urdiñeira, Orense ("Galicia no tempo" 1991: 140-141). Procedencia desconocida (Inventario, 1993: 128-129). Cilindro cerrado, forma ondulada a la cera perdida. Bronce Atlántico.

La forma ondulada de estos tres brazaletes se trabajó sobre el molde de cera antes de fundirlo. Se reconoce claramente la estructura de fundición en toda la superficie, lo que prueba el empleo de la cera perdida. El brazalete de Urdiñeira se decoró después con un punzón. La cera perdida los relaciona con el tipo V/E, lo mismo que la circularidad de los cilindros no puede excluir el empleo de una herramienta de eje rotativo. Por todo ello, estos tres brazaletes se diferencian del conjunto de aros ondulados en lámina fina de Arnozela, Braga (Inventario, 1993: 126-127) que están fabricados a forja y repujado.

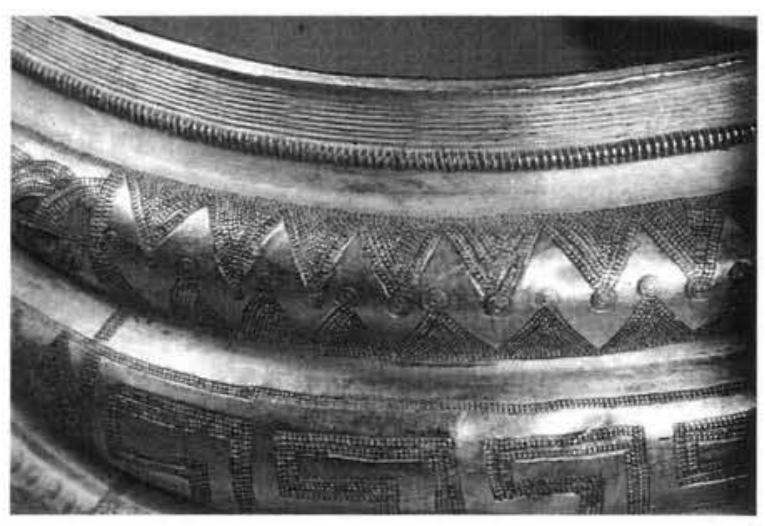

Lám. III. Detalle del borde superior, trabajado a torno, del brazalete de Lebuçao, Vila Real.

- Lebuçao, Vila Real (Severo, 1905-08). Cilindro cerrado, cera perdida, torno, forma ondulada por repujado, decoración a punzón. Cultura castreña.

El brazalete de Lebuçao puede considerarse una forma desarrollada de los brazaletes ondulados. Su forma cilíndrica se fundió a la cera perdida, y posiblemente el molde de cera fuera trabajado en un torno. La parte central del cilindro se repujó para hacer las ondulaciones, por lo que esta zona aparece más delgada; no así las zonas inferior y superior que permanecen macizas, con estrías trabajadas a torno (Lám. III). La decoración de la zona ondulada se realizó con un punzón especialmente elaborado para ello, y con el cuerpo del brazalete relleno de una sustancia que le diera consistencia.

- Dos ejemplares del Museo Nacional Soares dos Reis, Oporto, sin lugar de hallazgo (Pingel, 1992: 77, nº 338, lám. 107, 6). El Carambolo, Sevilla (Perea, 1991: 209). Cilíndricos, decoración de púas, soldadura. a) Transición Bronce Final, Primer Hierro; b) Orientalizante.

En este grupo se unen la tradición orfebre del Bronce Atlántico con la orientalizante de la Edad del Hierro. Los dos brazaletes del Museo Soares dos Reis, casi idénticos, se han relacio- 


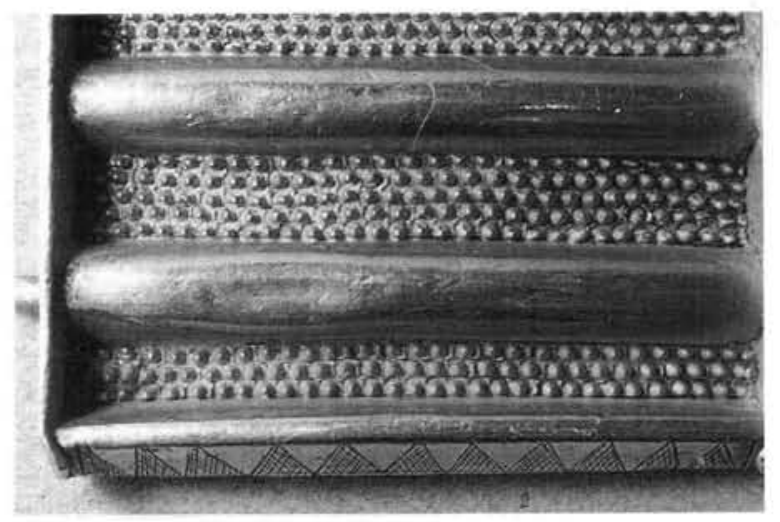

Lám. IV. Detalle del borde superior de uno de los brazaletes del Museo Soares dos Reis, Oporto, con decoración de púas e incisiones.

nado con el brazalete de Cantonha (Pingel, 1992: 77). Destacan por las siguientes particularidades: se han fabricado mediante soldadura de láminas, hilos y tiras con púas, parcialmente en hueco; las tiras con púas están fundidas a la cera perdida, como en los tipos V/E. El cuádruple cierre machihembrado es similar a los de piezas del Bronce Final, como el triple del torques de Alamo, Beja, y el doble del de Sagrajas, Badajoz. Se ha tomado el elemento formal de los torques y brazaletes macizos, de sección circular y decoración incisa, tipo Sagrajas/Berzocana, modificando la técnica y trabajando en

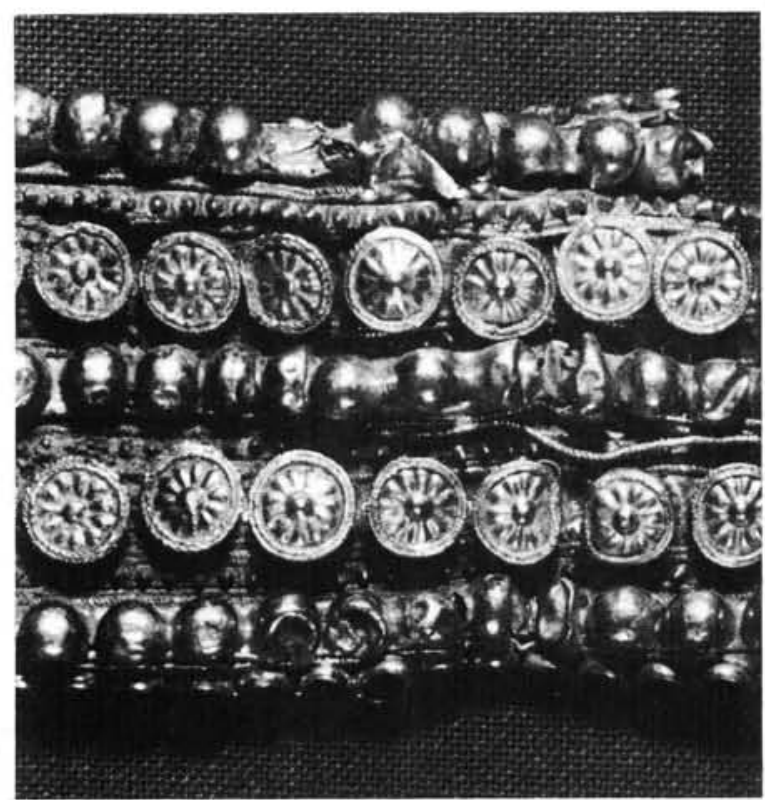

Lám. V. Placa, con púas, de El Carambolo, Sevilla. hueco para ahorrar material valioso; es en este aspecto en el que se puede establecer la comparación con Alamo. Finalmente, las molduras de los extremos, inferior y superior, estan decoradas con incisiones, y las tiras con púas se sueldan entre las molduras centrales (Lám. IV).

Algunas de las piezas del tesoro de $\mathrm{El} \mathrm{Ca-}$ rambolo, con decoración de púas realizada a la cera perdida (Lám. V), se pueden relacionar también con el tipo V/E. Ya Schüle (1960: 8081 , figs. 28,29 ) las había comparado con las púas del brazalete de Estremoz. Pero sobre todo, son los dos brazaletes cilíndricos los que aquí nos interesan. Se trata de brazaletes cilíndricos fabricados con técnicas de fundición de la Edad del Bronce, con soldadura de la Edad del Hierro, y rasgos del Bronce Final. La combinación con elementos de estilo orientalizante es un fenómeno nuevo.

\section{TORNO Y TALADRO EN LA ORFEBRERÍA DEL BRONCE FINAL ATLÁNTICO}

A partir de las huellas de trabajado y de uso, así como las diferentes texturas de la superficie que se observan con lupa binocular o incluso a ojo desnudo, podemos deducir los procesos de fabricación y herramientas empleadas sobre objetos metálicos.

En las piezas del tipo V/E no se observan huellas de soldadura, de cincelado o deformaciones plásticas realizadas con punzón, de manera que hay que rechazar las teorías propuestas hasta la fecha sobre la fabricación de los brazaletes cilíndricos. Por el contrario, se ven claramente huellas que apuntan a un proceso de fabricación complejo que incluye la técnica de la cera perdida y el empleo de instrumentos rotativos.

La reconstrucción de posibles herramientas rotativas se basa en diferentes analogías etnoarqueológicas e históricas, representaciones pictográficas y en la arqueología experimental.

\subsection{Instrumentos rotativos y su datación}

El descubrimiento del movimiento rotativo y la fuerza de giro fue la condición básica para el desarrollo de los instrumentos rotativos. Estos incluyen desde taladros, husos fusayolas, sellos cilíndricos, molinos, tornos, ruedas o carros hasta máquinas y motores (Horwitz, 1941). Ha- 
remos referencia a algunas de estas herramientas primitivas como ejemplo.

\section{La fusayola y el huso}

El desarrollo del hilado y los tejidos tuvo lugar durante el Neolítico. Los husos conservados son casi siempre de madera o hueso; la pieza giratoria de madera, barro o piedra. La varilla se pasa por la fusayola perforada. Estas suelen ser bicónicas, cónicas o discoides; se conservan sobre todo en barro.

Se conoce una primitiva representación de hilado, con el huso girando libremente, en la pintura mural de una tumba egipcia de la XII dinastía (2.000-1.800 a.C.) en Beni Hasan (Horwitz, 1941: 1802).

\section{Perforadores con eje vertical.}

Por el término "taladrar" entendemos la realización de orificios circulares y cilíndricos en materiales duros mediante el empleo de perforadores (o taladros) de movimiento rotativo. Es un proceso de lijado o molido por frotamiento que se diferencia del perforado con herramientas punzantes mediante tallado, raspado o golpeado. Las puntas para perforar, epipaleolíticas o neolíticas, de silex, así como las cuentas de hueso, bastones de cuerna o hachas de piedra perforadas, indican un conocimiento primitivo del proceso de taladro.

El taladro más simple es el palo de hacer fuego que gira entre las manos. Las representaciones más antiguas de perforadores, para ahuecar recipientes de piedra egipcios, se fechan en el III milenio a.C. (Childe, 1954: fig. 117).

Herramientas rotativas más evolucionadas son el taladro de accionamiento con arco y el de cuerda, cuyo eje de rotación presenta al menos un cojinete. Ambos consisten en un eje vertical en cuyo extremo se sujeta una punta para taladrar; en el extremo opuesto se dispone un revestimiento de sujeción que puede dirigirse con la mano, la boca o el pecho (Fig. 2a). Este revestimiente tiene generalmente forma de casquete y, además de servir de mango y protección del artesano contra el roce, sirve para hacer presión con el taladro sobre el objeto que se trabaja. El eje se pone en movimiento rotativo accionando la cuerda o el arco; en el primer caso se requieren dos personas; por el contrario, en el de arco sólo es necesario una. Además de numerosas analogías etnográficas, se conocen ejemplos pictográficos de taladros por arco en

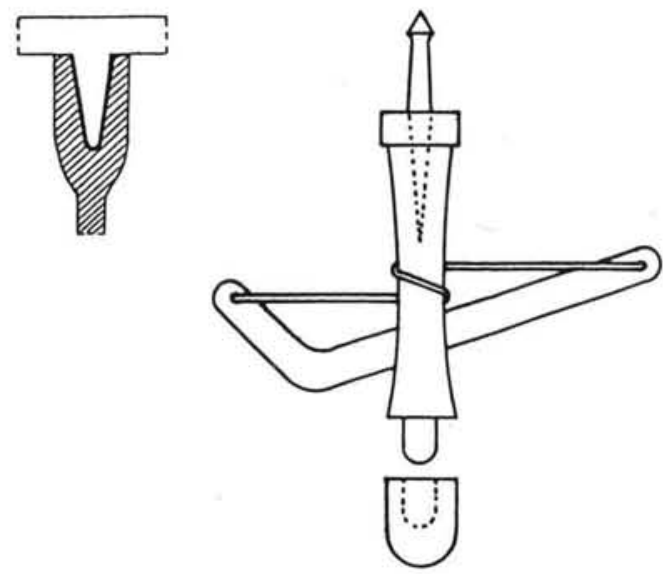

Fig. 2. a) Taladro o perforador de arco (según Untracht 1982). b) Herramienta de eje vertical con punta hueca.

tumbas egipcias y vasos griegos (Childe, 1954: fig. 112-115; Drescher, 1977-78; Born, 1989).

En la fabricación de nuestros brazaletes no se utilizó un taladro de eje vertical para las perforaciones; todas ellas, ovaladas o cuadradas, se realizaron previamente en el molde de cera, y más bien se utilizó una herramienta de eje vertical con punta hueca para el pulimento de las púas después de la fundición (Fig. 2b).

\section{Torno de eje horizontal}

El torno es una herramienta de eje horizontal que se sujeta en dos pies, como mínimo, y tiene un apoyo para el útil cortante que se maneja con la mano (Fig. 3). El eje se pone en movimiento mediante cuerda o arco, como en los perforadores, por lo que la rotación es igualmente alternativa, y se pueden trabajar materiales relativamente duros. El útil cortante, manejado a mano, se apoya contra el objeto que rota levantando virutas, por lo que este tipo de tra-

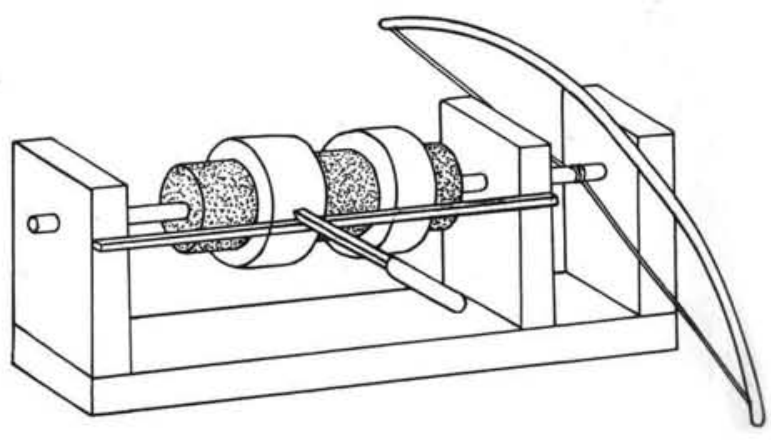

Fig, 3. Torno de eje horizontal. 
bajo sólo se puede efectuar girando el torno en una sola dirección. De esta manera el proceso tiene un momento activo y otro pasivo, ya que el torno de rotación continua sólo está documentado a partir de época romana (Mutz, 1972: 29-30).

Los objetos torneados son piezas cilíndricas, macizas o huecas, que se trabajan sobre un núcleo. Se sujetan al eje o forman por sí mismas un eje de rotación (Untracht, 1982: fig. 10, 57). La representación más antigua conocida hasta el momento de un torno procede del relieve de un tumba egipcia del siglo II a.C. (Lefebure, 1924: lám. 10); mediante una cuerda el trabajador lo pone en movimiento, mientras que otro sujeta una herramienta afilada contra el objeto (Fig. 4).

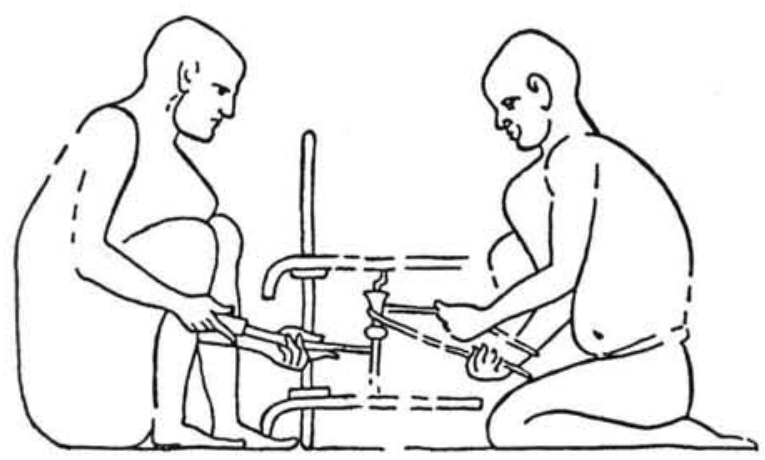

Fig. 4. Relieve egipcio con representación de trabajo a torno (según Lefebure, 1924).

Si transformamos este torno de manera que, en vez de un soporte en el extremo del eje, instalamos una punta de lijar, se podría utilizar como perforador horizontal, en cuyo caso ya no es necesaria la herramienta de cortar sino que es la propia pieza que se trabaja la que se acerca al elemento fijo que gira. Los pulidores de piedra, por ejemplo, utilizan este tipo de herramienta (Lefant, 1979: 238) que encontramos en ilustraciones etnográficas para la fabricación de recipientes.

La reconstrucción del perforador de rotación vertical durante el Neolítico (Drescher, 1976-77: fig. 49c) se considera el precursor del torno. Hasta la fecha, los tornos más antiguos de Europa central se datan en el Hallstatt (Drescher, 1980: 59).

Se ha comprobado que el torno se utilizaba para trabajar madera, marfil, ámbar, piedra, bronce y cera para la fundición de campanas medievales (Rieth, 1939-40; Drescher, 1984: 44-
55). En la reconstrucción de instrumentos rotativos en material perecedero no conservados, se han utilizado numerosas descripciones e ilustraciones de crónicas etnológicas, así como fuentes escritas medievales. El monje benedictino Theophilus Presbyter describió, en la primera mitad del siglo XII, los tornos accionados con cuerda y manivela (Brepohl, 1987: 181-182, fig. $61.1 \mathrm{y}$ 282-284, fig. 88.1).

Debido a que en tornería y alfarería se aprovecha el movimiento giratorio mediante un eje de rotación, en la bibliografía al uso se concluye frecuentemente que el torno de tornear ha evolucionado directamente del torno de alfarero. Aunque el torno de alfarero consiste en un eje vertical en cuyo centro se sitúa un disco horizontal (Childe, 1954: figs. 120, 121) y el producto obtenido es también un objeto con simetría de revolución, sin embargo y con razón, Mutz (1972: 17) considera que, desde el punto de vista tecnológico, no es problable esa evolución. Desde el punto de vista mecánico, ambas herramientas no tienen nada que ver, como tampoco tienen que ver su manejo y las propiedades físicas del material trabajado. Además, existen objetos torneados antes de la aparición del torno de alfarero.

El empleo del torno para el trabajo del metal no se había podido comprobar hasta hoy. Por primera vez se ha documentado en la fabricación de los brazaletes tipo $\mathrm{V} / \mathrm{E}$ en el suroeste de Europa durante el Bronce Final.

\subsection{La técnica de la cera perdida: problemas técnicos y soluciones}

La cera perdida es una técnica evolucionada que, frente a la fundición en molde de dos o más valvas, tiene la ventaja de permitir fundir objetos de formas complejas y paredes más finas. Un inconveniente sería que tras el vaciado hay que romper el molde; de manera que no es posible una producción en serie, sino vaciados individualizados. El principio sobre el que se basa la técnica es el siguiente: un modelo en cera del objeto deseado se recubre con varias capas de arcilla mezclada con material orgánico en la proporción adecuada; una vez seco se calienta la arcilla y se derrite la cera; en esta cavidad se vierte el metal fundido y una vez enfriado se rompe el molde y se limpia el objeto (Hunt 1985).

La cera perdida fué una técnica muy conocida en la Península durante el Bronce Final 
Atlántico; por ejemplo, entre los hallazgos calificados como "depósitos de fundidor"el del castro de Nossa Senhora da Guia, Baiôes, Viseu (Silva et alii., 1984) se encuentran excelentes trabajos en bronce fabricados a la cera perdida. Parece que en orfebrería se conocía la técnica con anterioridad; así, en el reciente estudio tecnológico que hicimos del tesoro de Caldas de Reyes, Pontevedra, se ha podido constatar que los cuerpos de las tres tazas de oro se fabricaron con esta técnica. Sin embargo, en los moldes de cera no se empleó el torno.

La fabricación de objetos con simetría de revolución para moldes en cera se conoce ampliamente por analogías etnográficas e históricas, por ejemplo, para la fundición de gongs (Mullens 1973-74), campanas (Drescher 1984: 44-55; Brepohl 1987: fig. 85,3), incensarios y recipientes de estaño (Brepohl, 1987: figs. 61,1; 85,3; 88,1). Aún hoy los fundidores de oro de Baulé y Ashanti, en Ghana y Costa de Marfil, testifican la fundición a la cera perdida de objetos de relieve complejo, en diferentes grosores y de excelente calidad (Fröhlich, 1981).

\subsection{Descripción técnica de la fabricación de brazaletes de oro}

Sobre la base de una amplia información interdisciplinar reconstruimos la fabricación de los brazaletes tipo $\mathrm{V} / \mathrm{E}$ de la siguiente manera.

\section{El modelo de cera}

La cera de abeja es un material relativamente blando, según la temperatura a la que se trabaje. Para su empleo en un torno es conveniente endurecerla; conocemos algunas recetas procedentes de textos antiguos y medievales para endurecerla mezclándola con carbón en polvo o colofonia.

La preparación del modelo en el torno es el primer paso. La forma básica -cilindro hueco y cerrado- comienza con la aplicación de una capa de cera en un núcleo de arcilla torneado sobre el eje (Lám. VI). Girando el torno, el orfebre corta el cilindro de cera en estrías antes de conformar las molduras (Fig. 5). Este modelo moldurado solamente se puede sacar del eje junto con el núcleo de arcilla. El interior de los brazaletes se deja generalmente liso.

En los tipos que presentan perforaciones, estas se realizan perforando sucesivamente la cera con puntas de metal calentadas, alisando después los espacios intermedios con raspadores.

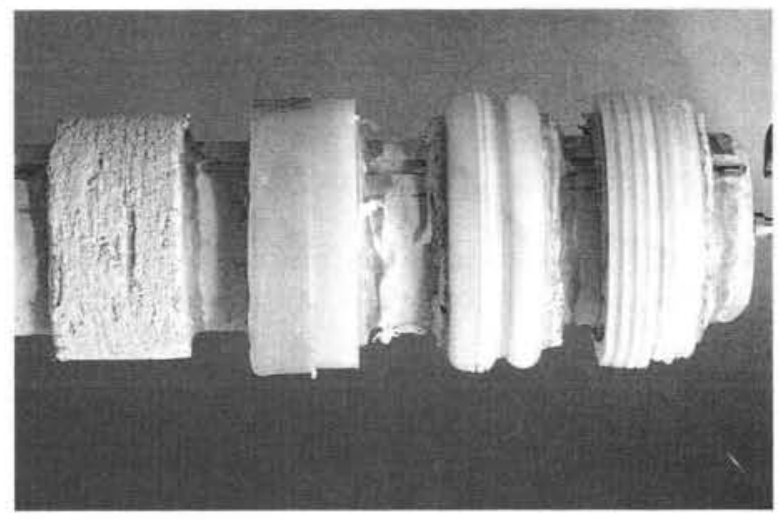

Lám. VI. Modelos de cera en distintas fases de trabajado sobre el eje de un torno horizontal.

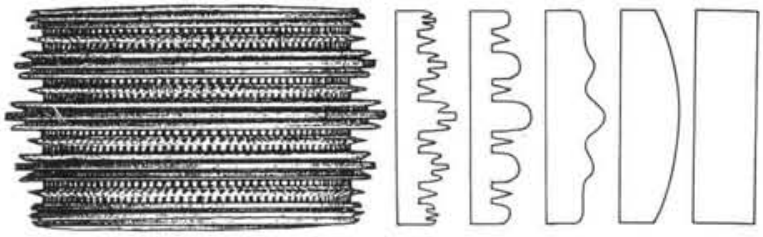

Fig. 5. Secciones sucesivas del modelo en cera durante el proceso de fabricación de un brazalete tipo Villena-Estremoz.

En los tipos con púas, el orfebre las realiza cortando perpendicularmente con una cuchilla caliente una de las molduras, formando así púas piramidales de base cuadrangular (Fig. 6). La cera se deforma plasticamente con facilidad empleando herramientas cortantes previamente calentadas; de este modo, con un punzón hueco de cavidad cónica, correspondiente al negativo de una púa de este tipo, se transforma la pirámide en cono (Fig. 6 y Lám. VII). El fragmento de brazalete procedente del M.A.N. ( $\mathrm{n}^{\circ}$ inv. 1962/7) muestra con claridad los cortes transversales realizados sobre la moldura.

Finalmente, se fijan sobre el modelo los canales y conos de fundición en cera. Lamentablemente, no se puede comprobar su situación original puesto que todos los brazaletes conservados han sido pulidos con posterioridad a este proceso.

\section{Molde y vaciado}

El paso siguiente consiste en la fabricación del molde de arcilla y el vaciado. Para ello se necesita en el taller un crisol, un fuelle con toberas y un horno de carbón.

El modelo de cera se recubre con varias capas de arcilla finamente molida y con desgra-

T. P., $51, \mathrm{n}^{\circ} 2,1994$ 

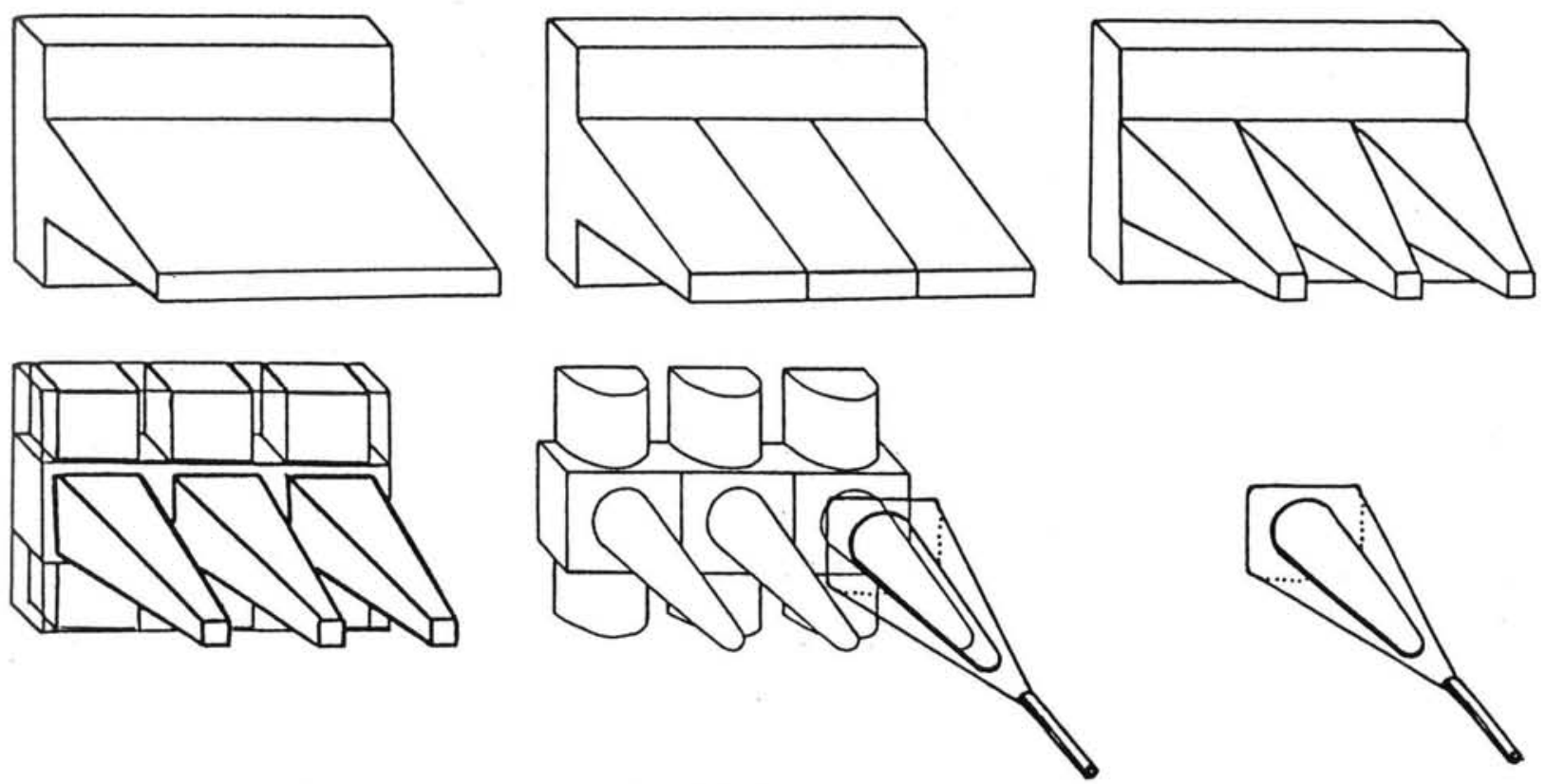

Fig. 6. Proceso de elaboración de las púas en el modelo de cera.

sante. Una vez seco el molde se calienta derritiéndose la cera, y cuando alcanza el rojo se vierte el oro fundido en la cavidad. Después de enfriarse, el molde se rompe y el objeto se limpia de restos de arcilla y se cortan los canales de fundición.

\section{Retocado y acabado}

Las rebabas de fundición, grietas o burbujas, se eliminan con cincel o piedra de lijar. En el torno se quitan las irregularidades de la superficie exterior del brazalete con ayuda de abrasivos. El empleo de fibras para aplicar estos abrasivos permite además pulir la superficie de estos objetos con simetría de revolución y topografía compleja, como el interior de las molduras.

Las púas se pulen con un perforador provisto de una broca con punta hueca; se ha comprobado la existencia de huellas de trabajado concéntricas no sólo en la superficie de las púas sino en su base.

Para el pulimento de los puentes de sección plano-convexa que quedan entre las perforaciones se pasan fibras por el interior de una y el exterior de la siguiente alternativamente. Enhebradas de esta manera, las fibras impregnadas de abrasivo pulen la superficie metálica frotando en uno y otro sentido. De este modo se pueden retocar también zonas de difícil acceso, donde están documentadas huellas de pulido.
La zona interna del brazalete aparece también pulida, sobre todo en aquella zona, más delgada, que se corresponde, por el exterior, con la línea de perforaciones; por el contrario, la zona correspondiente a las molduras, más gruesa, presenta todavía la superficie de fundición. Esto se explica por el comportamiento del metal al enfriarse tras el vaciado: la superficie interna del brazalete se contrae en función del correspondiente relieve externo. Los restos de esta superficie de fundición, a pesar del posterior pulido de acabado, son prueba fehaciente de que los brazaletes fueron vaciados.

\section{Los cortes}

Todos los brazaletes del tipo V/E estaban originalmente cerrados. Los 28 ejemplares de Villena se abrieron con posterioridad a su fabricación. Aquellos que presentan huellas de uso se abrieron de manera que cupiera el brazo. En los que no presentan esta huellas se puede observar perfectamente que los cortes se efectuaron "serrando", probablemente con fibras y arena. En algunas piezas se observa claramente la rebaba que dejó el corte realizado desde el exterior hacia el interior, sin respetar los elementos decorativos; el brazalete $\mathrm{n}^{\circ} 28$ (según numeración del catálogo de Soler 1965), presenta un corte, que pasa por el centro de una púa, con los bordes a ras. Hasta la fecha no se 
ha podido explicar por qué la mayor parte de los brazaletes aparecen abiertos, ¿quizá porque hubieran perdido su función simbólica y social? ¿se utilzarían desde la infancia y sólo cortándolos pudieron quitarse? ¿fueron chatarra que se transportaba más comodamente introduciéndose unos en otros, y cuyo valor se medía al peso? En el apartado donde se explica el modelo Villena se trata esta cuestión con más detalle.

\section{Reparaciones, defectos, huellas de uso}

El estudio técnico se realiza más fácilmente sobre objetos metálicos defectuosos o dañados. En el depósito de Villena se asocian brazaletes simples y complejos, de buena y mala calidad, defectuosos o con reparaciones. Por ejemplo, los brazaletes defectuosos n 25 y 27 (según numeración de Soler, 1965) presentan huellas de uso, de donde se deduce que las piezas defectuosas o reparadas no se desechaban. Lógicamente, las piezas de mala calidad se utilizaban, sin embargo, hay piezas excelentes que no presentan huellas de uso $\left(\mathrm{n}^{\circ} 28\right.$ y 29$)$.

En la pieza ${ }^{\circ} 27$ (Lám. VII) se reparó un defecto de fundición -una grieta en la parte externa- mediante una fundición adicional en la parte interna. Esta técnica sólo pudo aplicarse con anterioridad al conocimiento de la soldadura, como método de unión y arreglo. Anteriormente ya habíamos apuntado la existencia de esta técnica de unión en oro durante la Edad del Bronce en relación con el brazalete de Cantonha, Braga, y el torques macizo de Sagrajas, Badajoz.

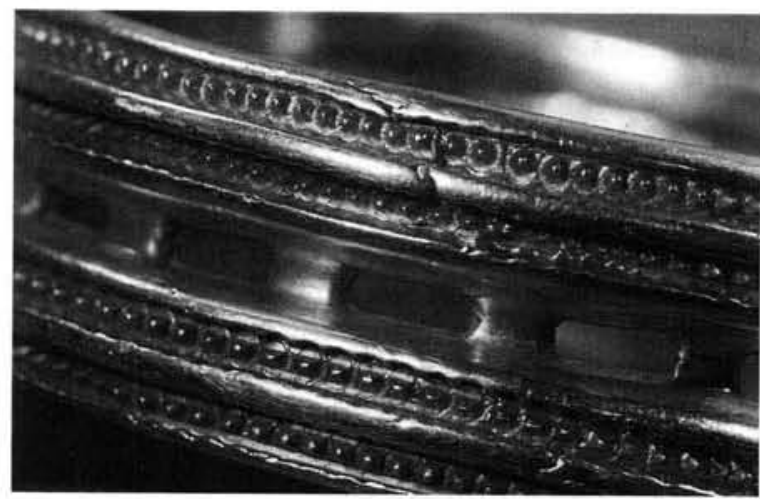

Lám. VII. Detalle de un brazalete de Villena con defecto de fundición. En la base de las púas se observa la huella circular de una herramienta de punta cónica hueca.

\section{Materiales y herramientas de orfebre}

Lamentablemente apenas conocemos útiles para el trabajo del metal en la Europa del suroeste, excepto los moldes. Sin embargo, no se puede concluir a partir de este dato que los objetos metálicos no fueran fabricados en la Península Ibérica. Se trata más bien de un vacío en la investigación. Mientras nuevas excavaciones no saquen a la luz este tipo de hallazgos, tendremos que basarnos en el estudio técnico de los objetos para poder reconstruir la historia de la metalurgia.

Aunque carezcamos de este tipo de información, es posible extraer conclusiones sobre las herramientas necesarias para la fabricación de un objeto en oro a través de las huellas de trabajado. Por la extraordinaria calidad de numerosas piezas prehistóricas deducimos que el orfebre sabía exactamente qué tipo de instrumento y en qué momento habría de utilizarlo, y que tenía a su disposición una amplia gama de herramientas.

Para la fabricación de los brazaletes tipo $\mathrm{V} / \mathrm{E}$ podemos suponer la siguiente lista de herramientas y materiales: cera para los modelos; útiles fabricados en cerámica como el horno, crisoles, toberas y arcilla acondicionada con material orgánico para los moldes; en bronce, cincel, punzón, puntas huecas, espátulas y leznas; en madera, el torno, el preforador de cuerda o arco con broca, quizá en piedra; abrasivos como arena, arcilla o ceniza y fibras para su aplicación.

\section{Arqueología experimental}

La Arqueología experimental se ocupa principalmente de la reconstrucción de los procesos técnicos en las culturas del pasado. Frecuentemente ocurre que conozcamos la herencia material de una cultura pero que desconozcamos los procesos, los usos, la función y el simbolismo de esos objetos. Para reconstruir técnicas perdidas en la actualidad es imprescindible recurrir a los modelos que nos proporcionan la experimentación práctica y las analogías funcionales de la etnoarqueología. La experimentación debe aplicarse de manera crítica, y en función del problema específico, ajustarse a las condiciones prehistóricas. Para ello es necesario el máximo de información técnica y la posibilidad de una práctica constatable. Sobre esta base práctica podremos desarrollar modelos que ha- 
gan probable un modo de fabricación del objeto entre varias posibilidades.

H. Drescher (1980: fig. 1.1) reconstruyó un torno manual de rotación alternativa según las indicaciones del monje Theophilus Presbyter (siglo XII) y las representaciones pictográficas egipcias (Fig. 4). El experimento que efectuamos para la fabricación de un modelo de brazalete tipo V/E se realizó con cera sobre capas de arcilla acondicionada con material orgánico en un eje de madera en un torno de rotación lenta. Primero se torneó un cilindro de arcilla, secada en cuero, mediante un cincel aguzado. Tras cubrirlo con capas de cera, se torneó en liso y se delimitaron los bordes (Lám. VI). Se siguió torneando hasta conseguir una sección plano-convexa y después una serie de molduras y surcos perfectamente paralelos y regulares (Fig. 5). Todo el proceso de torneado se realizó con ayuda de cuchillos, cinceles y puntas metálicas. El experimento respalda nuestra tésis de que los brazaletes tipo V/E se fabricaron a partir de modelos de cera torneada y se fundieron a la cera perdida.

\section{EL MODELO VILLENA (Fig. 7)}

El modelo que proponemos condensa la trayectoria temporal de los brazaletes tipo VillenaEstremoz. Es una propuesta a grandes rasgos de su biografía, que puede describirse como un largo proceso de mercantilización del objeto y se fundamenta en la contrastación del modelo Don-Mercadería (Appadurai, 1986) y el análisis de la evolución en los conceptos de "valor" y "demanda" (Perea, 1994 donde se explica la base teórica y conceptual). La finalidad última de este modelo es la verificación del mayor número de datos arqueológicos posible.

Partimos de una serie de presupuestos basados en el análisis detallado del depósito de Villena y materiales relacionados. Consideramos Villena un depósito heterogéneo y posiblemente diacrónico por las siguientes razones: a) diversidad funcional entre adornos, armas y vajilla; b) diversidad en el valor tecnológico de piezas de distintos tipos; c) diversidad de calidad técnica dentro de un mismo tipo, como los brazaletes; y d) distinto grado de conservación entre los distintos grupos funcionales. El valor tecnológico es función de la complejidad de la técnica por un lado, y del grado de control que
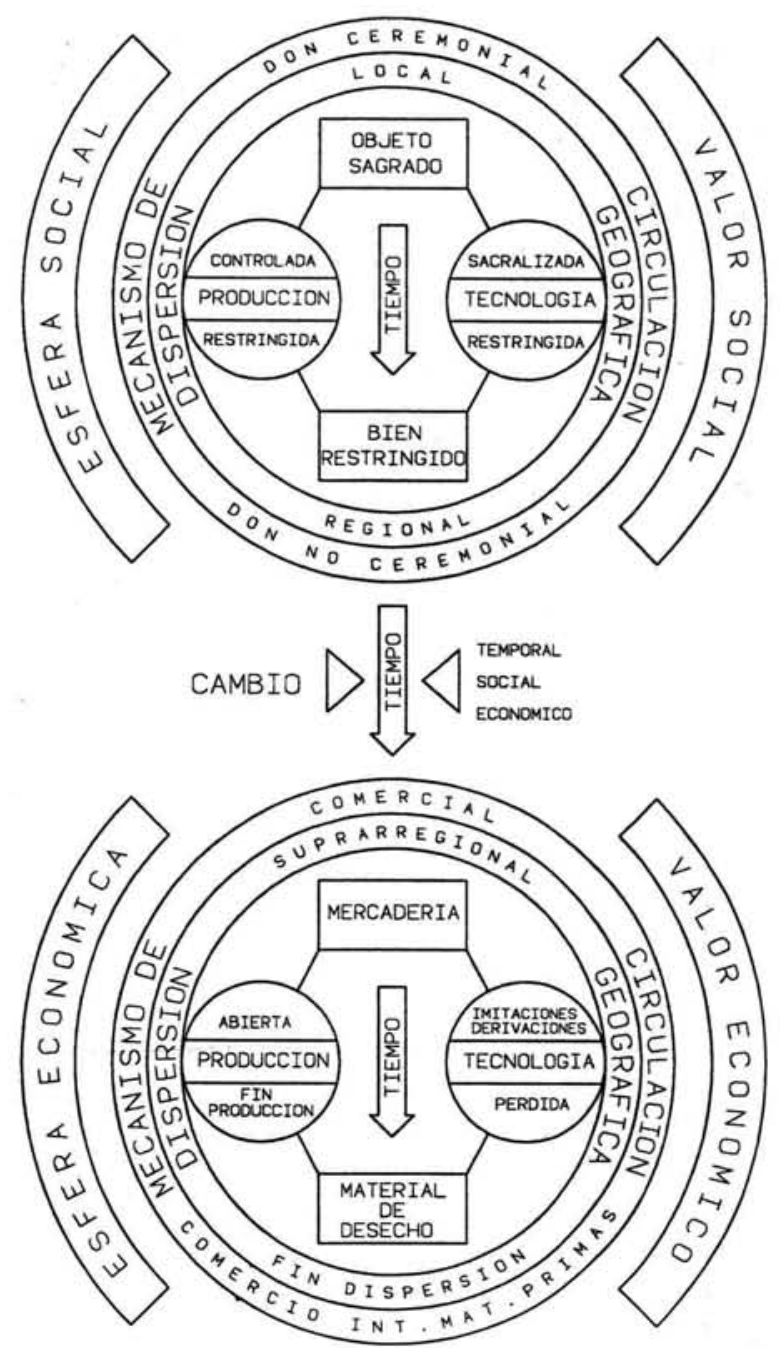

Fig. 7. El Modelo Villena

la sociedad ejerce sobre los artesanos que fabrican esos objetos tecnológicos por otro. En este sentido, creemos que los brazaletes poseen un alto valor tecnológico, al contrario que otras piezas procedentes del depósito, y son por ello producto de una tecnología punta controlada desde el poder.

El segundo presupuesto es la consideración de Villena como un único. No existe en la zona un patrón deposicional que avale tradición o costumbre de este tipo de acumulación o destrucción de riqueza. Por el contrario tendríamos que volvernos a la fachada atlántica para encontrar algo semejante; y allí, la norma son depósitos de oro con un peso que oscila entre $1.500 \mathrm{gr}$. y 2.800 gr., cifra muy alejada de los $9 \mathrm{~kg}$. de Villena. Centrándonos exclusivamente en los bra- 
zaletes tipo Villena-Estremoz, todos los hallazgos, excepto Villena con 28, se componen de un sólo ejemplar y a lo sumo dos, hecho que sí parece responder a un patrón deposicional intencionado. Finalmente, los 28 ejemplares de Villena, y gran parte de los hallazgos aislados, presentan un corte intencional a manera de destrucción simbólica del objeto; sólo algunos de los ejemplares de la fachada atlántica, como el de Estremoz, se han mantenido íntegros. Esos cortes no responden a ninguna solución funcional o técnica. Por todo ello, y lo anteriormente expuesto, creemos que Villena no tiene carácter de tesoro personal como se ha venido defendiendo, sino de material de desecho "para la exportación", por pérdida de su significado simbólico y cambio en su concepto de valor, bien por el paso del tiempo, traslado de ámbito cultural o transformaciones de tipo social y económico. El tipo diagnóstico son los brazaletes, cuya producción y consumo se desarrolló en la fachada atlántica peninsular, único ámbito tecnológico donde se daban las condiciones necesarias que permitieran el surgimiento de una tecnología punta.

Para la elaboración del gráfico de la figura 7 se han tenido en cuenta las tres variables del análisis económico: producción, distribución y consumo, que traducidas al lenguaje arqueológico serían: tecnología, mecanismos de circulación, y dispersión geográfica. La variable temporal se expresa en términos relativos.

El depósito de Villena, o lo que es lo mismo, la última etapa de consumo de los brazaletes tipo Villena-Estremoz, estaría en conexión con el circuito internacional de materias primas que se desarrolla a lo largo del siglo VIII a.C. entre la fachada atlántica y el Mediterráneo central (Ruíz-Gálvez, 1986; Sherrat y Sherrat, 1993). Su destino final hay que ponerlo en relación con el asentamiento de Peña Negra (Gonzalez Prats, 1993), Alicante, a pocos kilómetros de las desembocaduras de los ríos Segura y Vinalopó que debieron ser excelentes lugares de arribada. Según Ruíz-Gálvez (1989: 55; 1992) la ocultación de Villena se explica por la lucha del monopolio comercial con el Mediterráneo. Pero mientras la autora argumenta que el origen y fabricación de los brazaletes tuvo lugar en el levante, nosotros defendemos lo contrario por las razones ya expuestas. Villena era una zona estratégica y de paso obligado para el transporte por tierra, pero Cabezo Redondo era ya un lu- gar para el olvido, mientras que Peña Negra se encontraba en plena actividad artesanal y comercial.

\section{CONCLUSIONES (Fig. 8)}

A través del estudio tecnológico de un grupo de materiales de oro dispersos, hemos podido identificar y agrupar una corriente tecnológica que hasta la fecha no había sido aislada como tal (Armbruster, 1993a). La manifestación más espectacular de esta corriente han sido los brazaletes del depósito de Villena. La investigación tradicional había considerado este depósito como un conjunto de materiales sincrónicos, homogéneos y, para la mayoría de los autores, de origen local, lo que impidió su identificación y aislamiento de manera individualizada. Nos parece evidente, pero necesario señalar, que la existencia de un depósito no implica necesariamente homogeneidad tecnológica, cultural o cronológica de sus componentes. El despiezo de Villena es, en nuestra opinión, la clave para aproximarse a su mejor entendimento. En esta ocasión, nos hemos centrado exclusivamente en

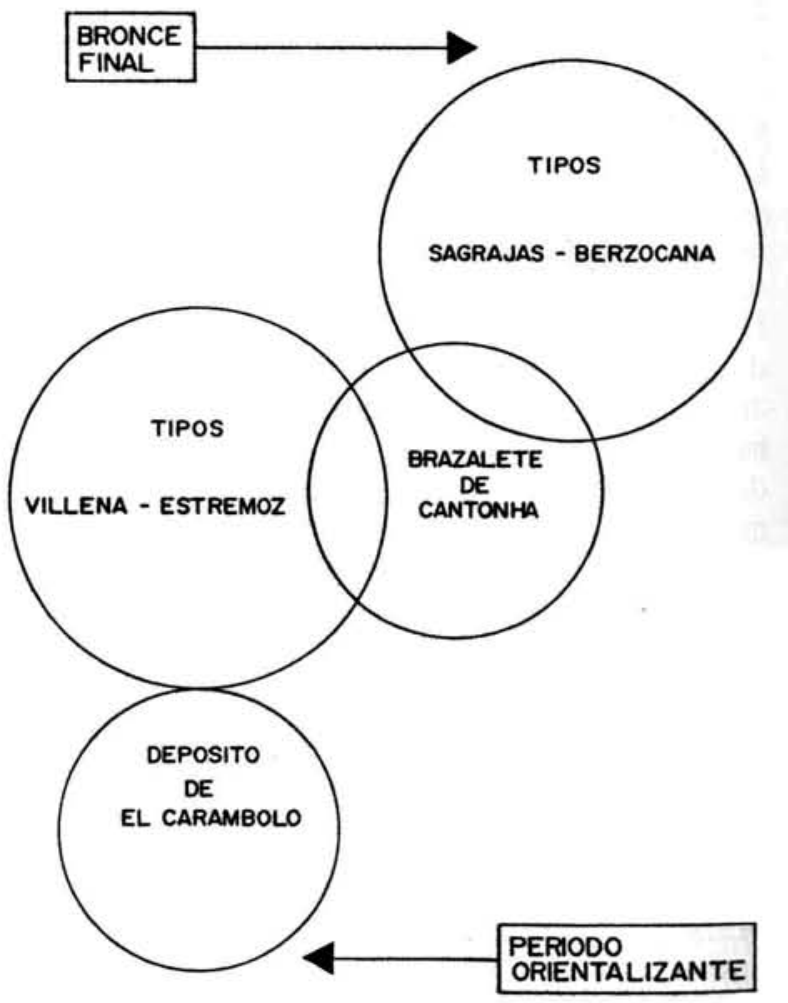

Fig. 8. Modelo estructural de la orfebrería durante el Bronce Final en la fachada atlántica. 
el análisis de los brazaletes por ser el material que presenta mayores retos interpretativos.

En el gráfico de la figura 8 planteamos una propuesta interpretativa sobre la orfebrería del Bronce Final en la fachada atlántica peninsular, en donde queda reflejada la cuestión cronológica y las interacciones tecnológicas.

Los círculos mayores representan dos grandes focos tecnológicos diferenciados y sin contactos aparentes, pertenecientes al entorno de la metalurgia atlántica; sus correspondientes manifestaciones arqueológicas son la "orfebrería tipo Sagrajas-Berzocana", definida en su momento por Almagro Gorbea (1974), y la "orfebrería tipo Villena-Estremoz", tal como la hemos definido en este estudio. Si partimos de la hipótesis que el material tipo Villena-Estremoz y el material Sagrajas-Berzocana han sido sincrónicos en determinado momento -y hay datos para pensar así- podemos plantear que la variabilidad entre ambos materiales se debe a las siguientes causas:

a) Primero, a su pertenencia a distintos grupos sociales con diferente grado de desarrollo tecnológico, al menos en el trabajo del oro. $\mathrm{La}$ base teórica de esta premisa es nuestra creencia en que determinada tecnología en particular, y los procesos tecnológicos en general, son producto de la capacidad de elección del hombre y del entorno social en el que se desenvuelve (Pfaffenberger, 1988). El conocimiento de la técnica del moldeado en su versión más sofisticada, la cera perdida, y la fabricación de moldes de cera a torno, utilizado igualmente en el acabado final de la pieza metálica, es prueba suficiente de la superioridad tecnológica del grupo Villena-Estremoz; entendiendo por superioridad la capacidad para resolver problemas formales y estructurales complejos.

b) Segundo, a una estrategia de identificación, entendida como la búsqueda de un lenguaje expresivo de la diferenciación entre dos grupos sociales a través de los signos de cultura material (Wiessner, 1984: 226-229; Conkey, 1989: 122-123; Pfaffenberger, 1992: 505), y en este caso concreto, a través de los símbolos de poder o estatus. La variabilidad formal y tecnológica entre los dos grupos de orfebrería del Bronce Final no puede ser explicada en términos de mutación, adaptación, evolución, o algún proceso aleatorio, por emplear el marco conceptual de la biología, sino que parece respon- der a una dinámica intencional en la generación de nuevas, o distintas, formas y procesos.

c) Tercero, a unos mecanismos de interacción social, incluido el intercambio de objetos y productos, que se desarrollan por diferentes canales. Solamente fuera del núcleo de origen de ambos círculos existe el dato de la integración de uno de los tipos en el otro: el brazalete de Cantonha (Braga). Esta pieza es la que aporta la base documental que permite defender la sincronía, aunque parece bien probado que la tecnología y los tipos Sagrajas-Berzocana tienen su origen en una tradición bastante más antigua dentro de la metalurgia atlántica (Almagro Gorbea, 1977). En este sentido, la tecnología Sagrajas-Berzocana sería el exponente de un producto maduro, mientras que VillenaEstremoz representaría la tecnología punta de un momento que perdura cuando la anterior ya ha desaparecido; sin embargo, hay que tener en cuenta que el elemento formal, la tipología Sagrajas-Berzocana, subsiste durante un tiempo empleando ya técnicas foráneas -piezas huecas, soldadura, etc.- propias de la transición al Primer Hierro, como demuestra, por ejemplo, el tesoro de Alamo, Beja (Inventario, 1993: 74-83).

En cuanto al problema cronológico, el límite inferior viene marcado por el depósito de El Carambolo. En este caso el círculo que lo representa no es secante sino tangente al tipo Villena-Estremoz porque no participa de todas sus características. En El Carambolo se integran rasgos tecnológicos, e iconográficos, de claro origen mediterráneo oriental -como las rosetas estampadas- con otros -como la fabricación de púas- con tecnología Villena-Estremoz. Este conjunto prueba que ha existido contacto y transmisión tecnológica directa.

La situación cronológica de la orfebrería Villena-Estremoz viene, entonces, enmarcada en su inicio y final por la orfebrería Sagrajas-Berzocana y por el fenómeno de la colonización fenicia. Sin embargo, carecemos de datos en términos absolutos, puesto que no existen piezas en contexto arqueológico fechable. El conjunto de El Carambolo ha sido fechado aproximadamente hacia finales del siglo VII a.C. por una de nosotras (Perea, 1991: 211-212), pero el estudio que ahora presentamos nos induce a elevar ligeramente esa fecha, puesto que no creemos en la larga perdurabilidad de la tecnología VillenaEstremoz, y menos aún dentro del ambiente de 
aculturación tecnológica que se produce en el Sur (Perea, 1991, 201-202). También con esta argumentación reivindicamos lo que ya en otra ocasión hemos defendido: la capacidad y alto nivel tecnológico del indígena, siempre cuestionada con respecto al colono fenicio, $\mathrm{y}$ la fabricación local e indígena de la llamada "orfebrería tartéssica" (Perea, 1991, 202).

Por el contrario, será en la zona más apartada del NW peninsular donde la tecnología de instrumentos rotativos y de la cera perdida perdure hasta fechas muy avanzadas, aunque las manifestaciones materiales conocidas pertenezcan ya a un ámbito cultural muy distinto, como el de la cultura castreña. En este sentido es importante concluir que la orfebrería castreña tiene su origen en la orfebrería del Bronce Final, al menos desde el punto de vista tecnológico -como demuestra el brazalete de Lebuçaoy sólo posteriormente se verá enriquecida por las innovaciones procedentes del sur peninsular, tanto como por las suyas propias.

La metalurgia del oro durante el Bronce Final es uno de los aspectos clave de la historia de la tecnología en la fachada atlántica peninsular a la hora de explicar fenómenos posteriores cuyo análisis requiere la perspectiva de larga duración. Esperamos haber contribuido a perfilar esa historia.

Darmstadt-Madrid, Noviembre 1993

\section{BIBLIOGRAFÍA}

Almagro Gorbea, M. (1974): "Orfebrería del Bronce Final en la Península Ibérica. El tesoro de Abía de la Obispalía, la orfebrería tipo Villena y los cuencos de Axtroki". Trabajos de Prehistoria, 31: 39-100.

- (1977): El Bronce Final y el Periodo Orientalizante en Extremadura. B.P.H., vol. XIV, Madrid.

Appadurai, A. (1986): The Social Life of Things. Commodities in Cultural Perspectives. Cambridge Univ. Press.

Armbruster, B.R. (1992): "Goldbergbau in Mali. Traditionelle Goldgewinnungsmethoden in Westafrika". Der Anschnitt, 44 (3): 70-79.

- (1993)a: "Instruments rotatifs dans l'orfèvrerie de l'âge du Bronze de la Péninsule Ibérique. Nouvelles connaissances sur la technique des bracelets du type Villena-Estremoz". Actas I Congresso de Arqueologia Peninsular, Porto: 265-279.

- (1993)b: "Etnoarqueología aplicada a la metalurgia del oro: el caso de Europa atlántica y Africa occidental". Trabajos de Prehistoria, 50: 113-126.
BIEK, L. (1963): Archaeology and the microscope. Londres. Blanco Freijeiro, A. (1957): "Origen y relaciones de la orfebrería castreña I y II". Cuadernos de Estudios Gallegos, 12: 5-28, 267-301.

Born, H. (1989): "Antike Bohrung in Metall". Acta Praehistorica et Archaeologica, 21: 117-130.

BREHPOL, E. (1987): Theophilus Presbyter und die mittelalterliche Goldschmiedekunst. Wien; Köln; Graz.

CARdozo, M. 1857: "Noticia de uma jóia antiga adquirida pelo Museu de Martins Sarmento". Revista de Guimarães, 67: 179-184.

- (1959): "Johalaria lusitana". Conimbriga, 1: 13-27.

ChILdE, G. 1954: "Rotary motion". En C. Singer E.J. Holmyard \& A.R. Hall (ed.): A history of technology, I. Oxford: 187-215.

CONKEY, M.W. (1989): "The use of diversity in stylistic analysis". En R.D. Leonard; G.T. Jones, Quantifying Diversity in Archaeology, 118-129. Cambridge Univ. Press.

Drescher, H. (1976/77): "Bohrer". Reallexikon der Germanischen Altertumskunde2. Bd. 3: 189-203.

- (1980): "Zur Technik der Hallstattzeit". En: Die Hallstattkutur, Frühform europäischer Einheit. Ausstellungskatalog, Steyr: 54-66.

- (1984): "Glockenfunde aus Haitabu". Berichte über die Ausgrabungen in Haitabu, 19: 9-62.

FröHLICH, M. (1981): "Zur Technik des Goldgusses bei den Ashanti (Ghana)". En E. Fischer \& H. Himmelheber, Das Gold in der Kunst Westafrikas. Museum Rietberg, Zürich: 43-58.

Galicia no Tempo (1991): Galicia no tempo. Catalogo de exposicion. Santiago de Compostela.

González Prats, A. (1993): "Quince años de excavaciones en la ciudad protohistórica de Herna (La Peña Negra, Crevillente, Alicante)". Sagvntvm, 26: 181-188

HoRwitz, H. (1941)a: Geräte mit Drehbewegung. CibaRundschau, 49, 1782-1793.

- 1941b: Die Entwicklung des Spinnens. Ciba-Rundschau, 49, 1795-1808.

Hunt, B. (1985): "The long history of lost wax casting, over five thousand years of art and craftsmanship". Gold Bulletin, 13 (2): 63-97.

Inventario do Museu Nacional de Arqueologia 1993: Colecçâo de Ourivesaria, $1^{\circ}$ volume, Do Calcolitico à Idade do Bronze. Lisboa

KALB, P. (1991): "Die Goldringe vom Castro Senhora da Guia, Baiões (Co. São Pedro do Sul), Portugal". Festschrift Wilhelm Schüle. Veröffentlichungen des vorgeschichtlichen Seminars in Marburg. Sonderband 6. Internationale Archäologie, 1: 185-200.

LARSEN, B. (1987): "SEM-identifcation and documentation of tool marks and surface textures on the Gundestrup cauldron". En Recent Advances in Conservation and Analysis of Artifacts. Institute of Archaeology, Londres: $393-408$.

Lefebure, M.G. (1924): Le Tombeau de Petosiris. III. Kairo.

Lenfant, J. (1979): "Bijouterie-joaillerie". Encyclopédie contemporaine des métiers d'art. Paris.

T. P., 51, nº 2, 1994 
Lowery, P. and Savage, R. (1972): "The technique of the decoration on a disc-butted axe from Romania". Proceedings of the Prehistoric Society, 38: 165-169.

Lowery, P.; Savage, R. and Wilkins, R. (1971): "Scriber, graver, scorper, tracer: notes on experiments in bronzeworking technique". Proceedings of the Prehistoric Society, 37: 167-182.

Maluquer, J. (1970): "Desarrollo de la orfebrería prerromana en la Peninsula Ibérica". Pyrenae, 6: 79-109.

Multens, W. (1973/74): “Java (Indonesien): Messingguß in 'verlorener Form'”. Begleitveröffentlichung von K. R. Wernhart. Publikationen zu wissenschaftlichen Filmen, Sektion Völkerkunde, Volkskunde 4, Institut für den wissenschaftlichen Film Göttingen: 94-102.

Mutz, A. (1972): Die Kunst des Metalldrehens bei den Römern. Basel.

Nicolini, G. (1990): Techniques des Ors Antiques. La bijouterie ibérique du VII au IV siècle. Picard, Paris.

OLSEN, S.L. (1988): "Introduction: applications of scanning electron microscopy to Archaeology". En S.L. Olsen (ed.): Scanning Electron Microscopy in Archaeology. B.A.R. Int. Series, 452: 3-7.

Parreira, R. y Pinto. C.V. (1980): Tesouros da Arqueologia Portuguesa no MNAE. Lisboa.

Perea, A. (1990): "Estudio microscópico y microanalítico de las soldaduras y otros procesos técnicos en la orfebrería prehistórica del sur de la Península Ibérica". Trabajos de Prehistoria, 47: 103-160.

- (1991): Orfebrería Prerromana. Arqueología del Oro. Comunidad de Madrid, Caja de Madrid.

- (1993): "Les premiers ateliers d'orfèvre dans la Péninsule Ibérique". En: Outils et Ateliers d'Orfèvres des Temps Anciens, Colloque Int., Saint Germain-en-Laye 1991: 23-28.

- (1994): "Proceso de mercantilización en sociedades premonetales". Archivo Español de Arqueología, en prensa.

Pfaffenberger, B. (1988): "Fetishized objects and humanized nature: toward an anthropology of technology". Man, 23: 236-252.

- (1992): "Social anthropology of technology". Annual Review of Anthropology, 21: 491-516.

Pingel, V. (1992): Die vorgeschichtlichen Goldfunde der Iberischen Halbinsel. Eine archäologische Untersuchung zur Auswertung der Spektralanalysen. MF 17, Berlin.
REINACH, S. (1912): “Un bracelet espagnol en or”. Revue Archéologique, 20:375-380.

RIETH, A. (1939/40): "Zur Technik antiker und prähistorischer Kunst: Das Holzdrechseln". Jahrbuch für prähistorische und ethnographische Kunst, 13/14: 85-107.

RUBIERA, M.J. (1985): Villena en las calzadas romana y árabe. Ayuntamiento de Villena, Alcoy.

Ruiz-GÁlveZ, M. (1986): "Navegación y comercio entre el Atlántico y el Mediterráneo a fines de la Edad del Bronce". Trabajos de Prehistoria, 43: 9-42

- (1989): “La orfebrería del Bronce Final. El poder y su ostentación". En El Oro en la España Prerromana, Monografía de Revista de Arqueología: 46-57.

- (1992): "La novia vendida: orfebrería, herencia y agricultura en la Protohistoria de la Península Ibérica”. Spal, 1:219-251

Russell, F. (1954): "O bracelete de Estremoz". Nummus, $2(6): 71-73$.

Schưle, W. (1960): "Probleme der Eisenzeit auf der Iberischen Halbinsel". Jahrbuch des Römisch-Germanischen Zentralmuseums Mainz, 7: 59-125.

- (1976): "Der Bronzezeitliche Funde von Villena (Alicante)". Madrider Mitteilungen, 17: 142-179.

Severo, R. (1905-08): "O tesouro de Lebução". Portugalia, 2 (1-4): 1-14.

Sherratt, A.; Sherratt, S. (1993): "The growth of the mediterranean economy in the early first millenium BC". En "Ancient Trade: New Perspectives", World Archaeology, 24 (3): 362-378.

Silva, A.C.F.; Silva, C.T. y Lopes, A.B. (1984): "Depósito de fundidor do final da Idade do Bronze do castro da Senhora da Guia (Baiões, São Pedro do Sil, Viseu)". Homenagen D. Domingos de Pinho Brandāo, Lucerna, Porto: 73-95.

Soler, J.M. (1965): El Tesoro de Villena. E.A.E., 36. Madrid.

- (1969): El Oro de los Tesoros de Villena. S.I.P., Serie Trabajos Varios 36, Valencia.

- (1987): Excavaciones Arqueológicas en el Cabezo Redondo (Villena, Alicante). Alicante.

TARradell, M. (1964): "Sobre el tesoro real de Villena", Saitabi, 14: 3-12.

Untracht, O. (1982): Jewelry, concepts and technology. London.

WIESSNER, P. (1984): "Reconsidering the behavioral basis for style: a case study among the Kalahari San". Journal of Anthropological Archaeology, 3: 190-234. 